\section{EPIDEMIOLOGY OF CATATONIA IN A LARGE DATASET}

1,2Jonathan P Rogers* ${ }^{*}{ }^{1,2}$ Thomas A Pollak, 'Nazifa Begum, ${ }^{1}$ Anna Griffin, ${ }^{1,2}$ Rashmi Patel, ${ }^{2}$ Megan Pritchard, ${ }^{2}$ Matthew Broadbent, ${ }^{1,2}$ Graham Blackman, ${ }^{1,2}$ Anna Kolliakou, ${ }^{1,2}$ Robert Stewart, ${ }^{1,2}$ Timothy RJ Nicholson ${ }^{3}$ Anthony S David. ${ }^{1}$ King's College London; ${ }^{2}$ South London and Maudsley NHS Foundation Trust; ${ }^{3}$ University College London

\subsection{6/jnnp-2019-BNPA.23}

Objectives/Aims Catatonia is an important neuropsychiatric disorder with a high morbidity and mortality. However, due to a perception that it is very infrequent and because of the acuity of the patients, it has remained poorly studied and research has often been confined to small groups. We aimed to establish the demographic, disease-related variables and blood-based biomarkers for catatonia in a large dataset.

Methods We used the Clinical Records Interactive Search (CRIS) system hosted at the NIHR Maudsley Biomedical Research Centre to search the clinical records for patients with catatonia. An initial free-text search was refined by use of a natural language processing app. The results of the app were validated by three of the authors, who included patients in the analysis only if a clinician had made a diagnosis of catatonia and two or more items of the Bush-Francis Catatonia Screening Instrument were in evidence. Demographics, diseaserelated variables and blood-based biomarkers could then be extracted for these patients and compared, where relevant, to non-catatonic psychiatric patients.

Results The natural language processing app extracted the records of 2766 patients with at least one mention of catatonia in their records. The majority of cases identified by the app could be validated by the researchers. A high proportion of patients had more than one episode of catatonia.

Full results will be available in time for the presentation. Conclusions This study demonstrates that catatonia is not very rare, even relying on clinician identification. The frequency of recurrence is interesting, as it suggests that catatonia might indicate an underlying trait, rather than merely a transient state.

\section{RELATIONSHIP BETWEEN INTEROCEPTION AND STRESS IN PATIENTS WITH FUNCTIONAL NEUROLOGICAL SYMPTOM DISORDER}

${ }^{1}$ Isobel A Williams, ${ }^{2}$ Markus Reuber, ${ }^{1}$ Liat Levita. ${ }^{1}$ Department of Psychology; ${ }^{2}$ Academic Neurology Unit, University of Sheffield

\subsection{6/jnnp-2019-BNPA.24}

Objectives/Aims Self-report studies of alexithymic traits in individuals with Functional Neurological Symptom Disorder (FND), suggest that emotion dysregulation in this population is characterised by an impaired ability to detect and identify their own emotions (identification impairments). ${ }^{1}$ This regulatory deficit might be particularly problematic for a patient group with an increased incidence of stressful life events relative to healthy controls, ${ }^{2}$ for whom the ability to regulate emotions might therefore be more crucial. Examining sensitivity to changes in physiological cues associated with emotional experience (interoception) is a way of assessing one aspect of participants' capacity to identify their own emotions. However, no studies have yet experimentally investigated how stress might interact with interoception in this population. Therefore, the aim of this study was to investigate patients' interoceptive sensitivity both at baseline and under stress.
Methods Twenty-six patients with FND and twenty-seven healthy controls performed the Heartbeat Detection Task (HBDT) preand post- stress-induction with the Cold Pressor Test. The HBDT is a behavioural paradigm, measuring participants' sensitivity to a physiological cue associated with emotional experience - the heartbeat. Participants also completed a self-report measure of emotion dysregulation (The Emotional Processing Scale-25) which includes a subscale capturing 'a detached experience of one's emotions due to poor emotional insight', and a measure of Major Depressive symptomology (The PHQ-9).

Results Relative to healthy controls, patients with FND performed more poorly on the HBDT both at baseline and following stress-induction $(p=0.032)$. Patients also reported greater impairments across all domains of the EPS-25 and higher scores on the PHQ-9 than healthy controls (both $\mathrm{p}<0.001$ ). Group differences on HBDT performance were not explained by group differences in age or depressive symptomology.

Conclusions Impaired HBDT performance suggests that patients with FND lack sensitivity to their heartbeat, both under 'normal' conditions and following stress-induction. Physiological cues (like the heartbeat) are an important source of interoceptive information for emotional experience, for example during stress. Our findings therefore represent a form of identification impairment that may contribute to stress-vulnerability in this population. Raised levels of self- reported impoverished emotional experience' corroborate the suggestion that patients with FND have difficulty identifying and understanding their emotions. These findings have direct implications for understanding and treating emotion dysregulation in FND.

\section{REFERENCES}

1. Williams IA, Levita L, Reuber M. Emotion dysregulation in patients with psychogenic nonepileptic seizures: A systematic review based on the extended process model. Epilepsy \& Behavior. 2018;86:37-48.

2. Ludwig L, Pasman JA, Nicholson T, Aybek S, David AS, Tuck S, et al. Stressful life events and maltreatment in conversion (functional neurological) disorder: systematic review and meta-analysis of case-control studies. The Lancet Psychiatry. 2018;5(4):307-20.

\section{ON BEING AUTOIMMUNE IN PSYCHIATRIC PLACES: 10 CHARACTERISTIC MENTAL STATE FEATURES IN PATIENTS WITH DEFINITE NMDAR-ANTIBODY ENCEPHALITIS}

\begin{abstract}
${ }^{1,2}$ Adam Al-Diwani, ${ }^{3}$ Ruth Linighan, ${ }^{3}$ Cheryl Perkins, ${ }^{4}$ Gail Critchlow, ${ }^{2,3,4}$ Belinda R Lennox, ${ }^{1,3} \mathrm{M}$ Isabel Leite, ${ }^{1,3}$ Sanjay Manohar, ${ }^{3}$ David Okai, ${ }^{1,3}$ Sarosh R Irani. ${ }^{1}$ Oxford Autoimmune Neurology Group, Nuffield Department of Clinical Neurosciences, University of Oxford, Oxford, UK; ${ }^{2}$ Department of Psychiatry, University of Oxford, Oxford, UK; ${ }^{3}$ Clinical Neurosciences, Oxford University Hospitals NHS Foundation Trust, Oxford, UK; ${ }^{4}$ Warneford Hospital, Oxford Health NHS Foundation Trust, Oxford, UK
\end{abstract}

10.1136/jnnp-2019-BNPA.25

Objectives/Aims NMDAR-antibody encephalitis frequently presents with psychiatric symptoms. However, new-onset mental illness does not usually receive detailed biomedical investigations. Yet, early diagnosis and treatment correlates with improved outcomes. Here we used detailed psychiatric phenotyping to explore the nature of mental state abnormalities in this immunologically-defined illness.

Methods Prospective and retrospective semi-structured interviews with patients, carers, and clinicians in five consecutive cases of definite NMDAR-antibody encephalitis (all female, median age $=20$ years, range $=16-30$, ovarian teratoma in 4). Weekly multi-disciplinary assessment using the Neuropsychiatric 